\title{
Desempenho agronômico de dez linhagens de soja-hortaliça
}

\author{
Hamilton César de O Charlo'; Roseli Pessoa²; Marina Funichello²; Renata Castoldi ${ }^{3}$; Leila T Braz \\ ${ }^{1}$ Instituto Federal de Educação Ciência e Tecnologia doTriângulo Mineiro (IFTM), R. João Batista Ribeiro 400, Mercês, $38064-790$ \\ Uberaba-MG; hamiltoncharlo@gmail.com; ${ }^{2}$ UNESP-FCAV, Depto. Fitossanidade, Rod. Prof. Paulo Donato Castellane s/n, 14884-900 \\ Jaboticabal-SP; ${ }^{3}$ UNESP-FCAV, Depto. Prod. Vegetal.
}

\section{RESUMO}

Objetivou-se avaliar o desempenho de dez linhagens de soja-hortaliça, em campo. O delineamento experimental adotado foi blocos ao acaso, com dez tratamentos (linhagens) e cinco repetições. Cada parcela experimental foi constituída por quatro linhas de plantio, com cinco metros de comprimento. Utilizou-se irrigação por aspersão. As linhagens avaliadas foram: JLM003; JLM004; JLM010; JLM018; JLM019; JLM024; JLM030; BR36; BR155 e BRS216. Avaliaram-se a precocidade média, altura de inserção da primeira vagem, número de vagens chochas, número de vagens com um grão, número de vagens com dois grãos, número de vagens com três grãos, massa fresca de vagens com um, dois e três grãos, massa fresca de 100 sementes, provenientes de vagens com um, dois e três grãos, massa de vagens não comerciais e produção de vagens comerciais por planta. Com base nos resultados obtidos, a linhagem JLM010 foi a mais indicada para a produção de soja-hortaliça por apresentar produção de grãos imaturos de $12,53 \mathrm{t} \mathrm{ha}^{-1}$ e maior massa fresca de 100 sementes.

\begin{abstract}
Agronomic performance of ten vegetable soybean strains, in Jaboticabal, Brazil

The objective was to evaluate the performance of ten vegetable soybean strains, in field. The experiment was arranged in randomized blocks with ten treatments (strain) and five replications. Plots comprised four rows measuring five meters in length. Sprinkle irrigation was used. The following strains were used: JLM003; JLM004; JLM010; JLM018; JLM019; JLM024; JLM030; BR36; BR155 and BRS216. The average earliness, insertion height of first pod, number of pods without grain, number of pods with one grain, number of pods with two grains, number of pods with three grains, fresh weight of pods with one, two and three grains, fresh weight of 100 seeds from pods with one, two and three grains, weight of no marketable pods and marketable production of pods per plant were evaluated. Results showed that the strain JLM010 is the best for vegetable soybean production, with total yield of immature grains of $12.53 \mathrm{t} \mathrm{ha}^{-1}$ and higher fresh weight of 100 seeds.
\end{abstract}

Keywords: Glycine max, strains, yield, edamame.

Palavras-chave: Glycine max, linhagens, produtividade, edamame.

\section{(Recebido para publicação em 30 de setembro de 2010; aceito em 2 de agosto de 2011)}

(Received on September 30, 2010; accepted on August 2, 2011)

$\mathrm{R}$ ecentemente introduzida no Brasil, a soja-hortaliça (edamame) (Glycine max) vem despertando grande interesse na população. A soja-hortaliça é comumente consumida no Japão, China, Coréia, Tailândia, Indonésia, Malásia, Filipinas, Cingapura, Nepal, Sri Lanka, Estados Unidos da América e Austrália (Mendonça \& Carrão Panizzi, 2003).

A soja-hortaliça é uma das hortaliças com maior potencial de crescimento de mercado no Brasil, pois pode ser uma alternativa natural de reposição hormonal, bem como um alimento de grande importância na alimentação humana, especialmente, pela presença das isoflavonas (Charlo et al., 2008). As isoflavonas têm efeitos semelhantes ao estrogênio, e isso favoreceu vários profissionais da saúde a aceitar as isoflavonas como uma alternativa à terapia de reposição hormonal. Além disso, existem também mecanismos não hormonais pelos quais as isoflavonas podem exercer efeitos fisiológicos in vivo (Messina, 2004), como por exemplo, regulação da atividade de proteínas (especialmente das tirosina quinases), regulação do ciclo celular e efeitos antioxidantes (Kurzer $\& \mathrm{Xu}, 1997)$.

Nos últimos anos a população tem buscado consumir alimentos mais saudáveis e que tragam benefícios à saúde. Neste sentido, tem crescido a demanda por produtos com maior valor agregado, como é o caso da soja-hortaliça. Com base nisto, têm sido introduzidas e desenvolvidas, no Brasil, cultivares de soja com características especiais para o consumo in natura, podendo ser chamada de soja-verde, soja-hortaliça, ou edamame (quando submetida à cocção em água e sal). Quando destinadas para este fim, as sementes são consumidas imaturas e ocupam 80 a $90 \%$ da largura das vagens, o que corresponde ao estádio $\mathrm{R}_{6}$ (Konovsky \& Lumpkin, 1990). O Japão, a China, a Coréia e o Taiwan são os principais produtores e consumidores desse tipo de alimento (Carrão-Panizzi, 2006). Além disso, contém alto teor de proteínas $(13 \%)$, reduzido teor de óleo $(5,7 \%)$, não possui colesterol e gordura hidrogenada e detem minerais, fósforo, cálcio e vitaminas $B_{1}$ e $B_{2}$ (Shanmugasundaram \& Yan, 2004).

As cultivares de soja-hortaliça devem apresentar características especiais, diferentes da soja comum, como sementes graúdas com alto teor de proteína, coloração clara do hilo; ausência de enzimas lipoxigenases (AL), conferindo sabor mais suave aos produtos; teor reduzido do inibidor de tripsina Kunitz (KI), o que permite a redução de tratamento térmico e dos custos de processamento e tamanho, coloração e textura de sementes ideais para produção de natto (alimento fermentado japonês). Entre as cultivares desenvolvidas para esse fim e que apresentam algumas das características citadas, destacam-se: BR 36 (QO); BRS 155 (KR); BRS 213 (AL); BRS 216 (PN); IAC PL-1 (QO); 
UFVTN 101 (AL); UFVTN 102 (AL); UFVTN 103 (AL); UFVTN 104 (AL); UFVTN 105 (AL), e UFVTNK 106 (AL, KR) (Embrapa, 2003).

Em 2008, tradicionais produtores de hortaliças do Cinturão Verde de São Paulo iniciaram cultivos de soja-hortaliça, visando a atender nichos específicos de mercado na cidade de São Paulo, porém, estes produtores utilizaram sementes de cultivares importadas da China, as quais são bem adaptadas às condições brasileiras. No Brasil, existem muitas cultivares de soja com potencial de uso como soja-hortaliça, porém apresentam produtividade muito variada, dependendo das condições em que são cultivadas, como solo, clima, época de plantio e densidade de plantio.

Diante do exposto, o presente trabalho teve por objetivo avaliar dez linhagens com características de soja-hortaliça, quanto ao seu desempenho agronômico.

\section{MATERIAL E MÉTODOS}

O experimento foi conduzido em campo, de 20/11/2008 a 02/04/2009, em área experimental do Setor de Olericultura e Plantas Aromático-Medicinais, pertencente à UNESP, Campus de Jaboticabal-SP (21 ${ }^{\circ} 14^{\prime} 05^{\prime}$ 'S, 48 $17^{\circ}$ '09' 'W, altitude de $614 \mathrm{~m}$ ). O clima, segundo a classificação de Köppen, é do tipo Aw com transição para Cwa $\left(\right.$ Volpe $\left.^{1}\right)$, e o solo da área experimental foi classificado como sendo Latossolo eutroférrico (Embrapa, 1999).

As sementes utilizadas foram oriundas da Embrapa-Hortaliças. Foram avaliadas as linhagens JLM003; JLM004; JLM010; JLM018; JLM019; JLM024; BR36; BR155 e BRS216. O delineamento experimental adotado foi blocos ao acaso, com dez tratamentos (linhagens) e cinco repetições por tratamento. Cada parcela experimental foi constituída por quatro linhas de 5,0 $\mathrm{m}$ de comprimento, com $15 \mathrm{~cm}$ entre plantas e $60 \mathrm{~cm}$ entre linhas, sendo consideradas para avaliação 30 plantas por parcela, das duas linhas centrais.
Devido ao intenso ataque de pombos em plantios anteriores com semeadura direta, optou-se pela formação das mudas, utilizando-se bandejas de poliestireno expandido, com capacidade para 128 células, preenchidas com substrato comercial Plantmax Hortaliças ${ }^{\circledR}$. Colocou-se uma semente por célula, já inoculada na proporção de 500 gramas de inoculante, que contêm Bradyrhizobium spp, para cada $50 \mathrm{~kg}$ de sementes.

Para a instalação do experimento, o solo foi amostrado na profundidade de 0-20 cm, sendo a amostra enviada ao Laboratório de Fertilidade de Solo para análises químicas, com os seguintes resultados: $\mathrm{pH}$ em $\mathrm{C}_{\mathrm{a}} \mathrm{Cl}_{2}=5,3 ; \mathrm{MO}=18,0 \mathrm{~g}$ $\mathrm{dm}^{-3} ; \mathrm{P}_{\text {resina }}=64,0 \mathrm{mg} \mathrm{dm}^{-3} ; \mathrm{K}=3,1 \mathrm{mmol}_{\mathrm{c}}$ $\mathrm{dm}^{-3} ; \mathrm{Ca}=62,0 \mathrm{mmol}_{\mathrm{c}} \mathrm{dm}^{-3} ; \mathrm{Mg}=30,0$ $\mathrm{mmol}_{\mathrm{c}} \mathrm{dm}^{-3} ; \mathrm{H}+\mathrm{Al}=22,0 \mathrm{mmol}_{\mathrm{c}} \mathrm{dm}^{-3}$; $\mathrm{SB}=70,1 \mathrm{mmol}_{\mathrm{c}} \mathrm{dm}^{-3} ; \mathrm{T}=99,6 \mathrm{mmol}_{\mathrm{c}}$ $\mathrm{dm}^{-3} ; \mathrm{V} \%=67$.

As mudas foram transplantadas aos dez dias após a semeadura, em solo anteriormente preparado e adubado, conforme os resultados da análise do solo e a recomendação de Raij et al. (1997) para a cultura da soja. Para tanto, foram aplicados em adubação de plantio, $40 \mathrm{~kg} \mathrm{ha-1}$ de $\mathrm{P}_{2} \mathrm{O}_{5}$, e $60 \mathrm{~kg} \mathrm{ha}^{-1}$ de $\mathrm{K}_{2} \mathrm{O}$, utilizando superfosfato simples e cloreto de potássio como fonte, respectivamente, sendo que o $\mathrm{K}_{2} \mathrm{O}$ foi parcelado, aplicando-se metade da dose na adubação de plantio e a outra metade em adubação de cobertura, realizada 30 dias após o transplante.

Utilizou-se irrigação por aspersão, quando necessário para manter o solo sempre úmido e adequado para um melhor desenvolvimento da cultura. O controle de plantas invasoras foi realizado através de capina manual, mantendo-se a cultura no limpo durante todo o ciclo de cultivo.

Para o controle de pragas e doenças, foi adotado o manejo conforme a ocorrência do agente, inseto ou patógeno na área experimental, e de acordo com recomendações técnicas do produto químico utilizado. Os produtos utilizados foram: Metamidophos (0,5 L/ha), Difenoconazole (150 mL/ha) e Azoxystrobin
+ Ciproconazole (300 mL/ha). Deve-se ressaltar que esses produtos são registrados para a cultura da soja e foram utilizados para controlar as seguintes pragas: vaquinha (Megascelis SP.), percevejos (Nezara viridula, Piezodorus guildini e Euschistos heros) e lagarta-da-soja (Anticarsia gemmatalis) e doenças: oídio (Microsphaera diffusa) e ferrugem asiática (Phakopsora pachyrhizi).

As colheitas foram realizadas quando as vagens estavam em estádio reprodutivo $\mathrm{R}_{6}$, tendo como referência a escala de Fehr \& Caviness, adaptada por Costa \& Marchezan (1982). Com a ajuda de uma tesoura, as plantas foram cortadas acima da superfície do solo, e levadas para o laboratório, onde as vagens foram retiradas.

Foram avaliados a precocidade (dias da semeadura até a colheita); altura de inserção da primeira vagem (mensurada em centímetros); número de vagens chochas; números de vagens com 1 grão; número de vagens com 2 grãos; número de vagens com 3 grãos; número total de vagens comerciais; massa fresca de 100 grãos de vagens com 1, 2 e 3 grãos (mensurada em gramas por 100 grãos); massa de vagens não comerciais (mensuradas em gramas por planta); massa fresca de vagens com 1, 2 e 3 grãos (mensuradas em gramas por planta); massa fresca total de vagens comerciais (mensurada em gramas por planta) e produtividade total estimada por hectare (mensurada em $\mathrm{t} / \mathrm{ha}$ ), onde considerou-se para o cálculo, a massa fresca de 100 grãos, o número de vagens por planta, o número de grãos por vagem e o número de plantas por hectare.

Os dados obtidos foram submetidos à análise de variância, e as médias, agrupadas pelo Teste de Scott-Knott (Scott \& Knott, 1974), em nível de 5\% de probabilidade, utilizando o programa Agroestat (Sistema para Análises Estatísticas de Ensaios Agronômicos), desenvolvido pelo Prof. Dr. José Carlos Barbosa e Walter Maldonado Junior, UNESP- FCAV, Campus Jaboticabal.

\section{RESULTADOS E DISCUSSÃO}

As linhagens JLM010 (97 dias), JLM019 (97 dias) e BRS155 (97 dias) 
Tabela 1. Médias das características precocidade (PREC), altura de inserção da primeira vagem $(\mathrm{ALT} 1 \mathrm{~V})$, número de vagens chochas $(\mathrm{NVCH})$, número de vagens com um grão (NV1G), número de vagens com dois grãos (NV2G), número de vagens com três grãos (NV3G) para dez linhagens de soja-hortaliça [averages of cycle length (PREC), height of insertion of first pod (ALT1V), number of pods without grain $(\mathrm{NVCH})$, number of pods with one grain (NV1G), number of pods with two grains (NV2G), number of pods with three grains (NV3G) of ten strains of vegetable soybean]. UNESP, Jaboticabal, 2009.

\begin{tabular}{lrrrrrr}
\hline Linhagens & $\begin{array}{r}\text { PREC } \\
(\text { dias })\end{array}$ & $\begin{array}{r}\text { ALT1V } \\
(\mathbf{c m})\end{array}$ & NVCH & NV1G & NV2G & NV3G \\
\hline BR36 & $104 \mathrm{c}$ & $7,55 \mathrm{~b}$ & $2,10 \mathrm{~b}$ & $15,60 \mathrm{c}$ & $64,00 \mathrm{a}$ & $19,16 \mathrm{c}$ \\
JLM010 & $97 \mathrm{~d}$ & $9,10 \mathrm{~b}$ & $0,63 \mathrm{~b}$ & $13,99 \mathrm{c}$ & $44,87 \mathrm{a}$ & $7,12 \mathrm{c}$ \\
JLM030 & $104 \mathrm{c}$ & $7,76 \mathrm{~b}$ & $1,80 \mathrm{~b}$ & $13,53 \mathrm{c}$ & $54,13 \mathrm{a}$ & $10,33 \mathrm{c}$ \\
JLM019 & $97 \mathrm{~d}$ & $7,41 \mathrm{~b}$ & $3,26 \mathrm{~b}$ & $8,50 \mathrm{c}$ & $58,80 \mathrm{a}$ & $29,20 \mathrm{~b}$ \\
BRS155 & $97 \mathrm{~d}$ & $11,49 \mathrm{a}$ & $6,23 \mathrm{a}$ & $14,66 \mathrm{c}$ & $53,30 \mathrm{a}$ & $40,40 \mathrm{a}$ \\
JLM018 & $111 \mathrm{~b}$ & $12,52 \mathrm{a}$ & $6,06 \mathrm{a}$ & $29,86 \mathrm{a}$ & $48,96 \mathrm{a}$ & $3,93 \mathrm{c}$ \\
JLM003 & $120 \mathrm{a}$ & $11,23 \mathrm{a}$ & $2,66 \mathrm{~b}$ & $17,36 \mathrm{c}$ & $42,70 \mathrm{a}$ & $8,10 \mathrm{c}$ \\
JLM020 & $111 \mathrm{~b}$ & $11,04 \mathrm{a}$ & $7,03 \mathrm{a}$ & $33,70 \mathrm{a}$ & $55,86 \mathrm{a}$ & $5,93 \mathrm{c}$ \\
JLM004 & $120 \mathrm{a}$ & $15,76 \mathrm{a}$ & $2,56 \mathrm{~b}$ & $13,93 \mathrm{c}$ & $22,70 \mathrm{a}$ & $5,16 \mathrm{c}$ \\
JLM024 & $111 \mathrm{~b}$ & $8,16 \mathrm{~b}$ & $4,03 \mathrm{~b}$ & $23,17 \mathrm{~b}$ & $47,65 \mathrm{a}$ & $12,63 \mathrm{c}$ \\
\hline Teste F & $38,59 * *$ & $15,24 * *$ & $9,24 * *$ & $9,32 * *$ & 4,09 NS & $17,25 * *$ \\
CV (\%) & $2,30 \%$ & $11,76 \%$ & $13,54 \%$ & $24,55 \%$ & $19,83 \%$ & $25,24 \%$ \\
\hline
\end{tabular}

Médias seguidas pelas mesmas letras, na coluna, não diferem significativamente, em nível de $5 \%$ de probabilidade, pelo teste de agrupamento de médias de Scott-Knott (1974); ${ }^{\text {NSNão }}$ significativo; ${ }^{*}$ significativo a $1 \%$ de probabilidade (means followed by the same letter in column are not different through the means grouping test of Scott-Knott (1974) at 5\%;

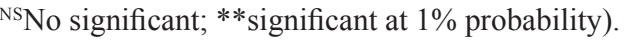

foram as mais precoces (Tabela 1), ou seja, as primeiras que tiveram suas vagens mais rapidamente no ponto de colheita. As linhagens mais tardias foram JLM003 e JLM004 (ambas com 120 dias). De acordo com Embrapa (2003) o ciclo de cultivares de soja pode variar de acordo com local, a época de cultivo e a latitude de cada região. Os resultados obtidos no presente trabalho corroboram com o trabalho de Castoldi et al. (2011), que avaliaram cinco linhagens de soja-hortaliça, quanto às características agronômicas, funcionais e antinutricionais, e verificaram números de dias para colheita variando de 98 dias (JLM010) a 138 dias (JLM003 e JLM004), classificando-as em dois grupos de maturação: precoce (JLM010, JLM024 e JLM030) e semitardio (JLM003 e JLM004).

No Brasil, as colheitadeiras não são preparadas para colher grãos com alto teor de umidade (acima de 14\%), porém é de fundamental importância selecionar plantas com alta inserção de vagens, facilitando a adaptação de novas máquinas para colheita. As linhagens que expressaram as maiores médias de altura de inserção de vagens foram JLM004 (15,76 cm), JLM018 (12,52), BRS155 (11,49 cm), JLM003 (11,23 $\mathrm{cm})$ e JLM020 $(11,04 \mathrm{~cm})$ podendo, possivelmente, ocorrer menores perdas utilizando a colheita mecanizada.

Com relação ao número de vagens chochas por planta (Tabela 1), verificou-se que BRS155 (6,23), JLM018 $(6,06)$ e JLM020 $(7,03)$ revelaram as maiores médias de número de vagens chochas, ou seja, vagens sem nenhum interesse econômico. Porém, de maneira geral, apesar de ter ocorrido diferença estatística entre as linhagens, todas apresentaram baixa porcentagem de vagens chochas.

Segundo Konovsky \& Lumpkin (1990), as cultivares utilizadas como soja-hortaliça devem ter preferencialmente vagens com dois ou mais grãos por unidade. Portanto, devem-se selecionar linhagens que apresentam menor quantidade de vagens com um grão, e maior quantidade de vagens com dois e três grãos.
Para a média de número de vagens com um grão, os maiores valores foram observados para JLM020 $(33,70)$ e JLM018 $(29,86)$, diferindo estatisticamente das demais linhagens, as quais detiveram menor número de vagens com um grão.

Para vagens com dois grãos, não foram observadas diferenças significativas entre as linhagens. Já para número de vagens com três grãos, observou-se maior valor para BRS155 $(40,40)$, seguido da linhagem JLM019 $(29,20)$. Isso se deve ao fato de que BRS155 foi melhorada para a produção de soja-grão, e nos processos de seleção e melhoramento, os pesquisadores visaram grande número de sementes por vagem. No entanto, esta linhagem também pode ser utilizada como soja-hortaliça por apresentar boa palatabilidade.

Verificou-se na Tabela 2 que JLM010, JLM030, JLM003 e JLM004 revelaram menor número total de vagens comerciais por planta. Porém, este resultado isoladamente não é suficiente para descartar uma linhagem de soja-hortaliça, pois pode haver efeito compensatório do tamanho da semente de determinada linhagem, o que é ideal para este segmento de soja, ou seja, plantas com número reduzido de vagens e com grãos bem grandes.

Quanto à massa fresca de 100 grãos, verificou-se que tanto para vagens com um, dois ou três grãos, JLM010 expressou maiores valores, podendo-se concluir que possui grãos mais graúdos e mais aceitáveis para consumo como soja-hortaliça. Castoldi et al. (2008), ao comparar cinco linhagens de soja-hortaliça, também verificaram superioridade da linhagem JLM010 em relação às demais, para a característica massa fresca de 100 grãos.

Em detrimento do maior número de vagens não comerciais em JLM20, BRS155 e JLM018, observaram-se maiores médias de massa de vagens não comerciais nestas linhagens, verificando-se médias para esta característica respectivamente de: 2,$35 ; 1,87$ e 1,82 g/planta.

Com relação à massa fresca de vagens com um grão não se detectou diferença significativa entre as linhagens avaliadas, provavelmente em função do 
Tabela 2. Médias das características número total de vagens comerciais (NTVC), massa fresca de 100 grãos de vagens com um grão $\left(M_{100} \mathrm{GV1G}\right)$, massa fresca de 100 grãos de vagens com dois grãos $\left(\mathrm{M}_{100} \mathrm{GV} 2 \mathrm{G}\right)$, massa fresca de 100 grãos de vagens com três grãos $\left(\mathrm{M}_{100} \mathrm{GV} 3 \mathrm{G}\right)$, massa de vagens não comerciais (MVNC) e massa fresca de vagens com um grão (MV1G) para dez linhagens de soja-hortaliça [average total number of marketable pods (NTVC), fresh weight of 100 grains of pods with one grain $\left(M_{100} G V 1 G\right)$, fresh weight of 100 grains of pods with two grains $\left(\mathrm{M}_{100} \mathrm{GV} 2 \mathrm{G}\right)$, fresh weight of 100 grains of pods with three grains $\left(\mathrm{M}_{100} \mathrm{GV} 3 \mathrm{G}\right)$, weight of no-marketable pods (MVNC) and fresh weight of pods with one grain (MV1G) for ten strains of vegetable soybean]. UNESP, Jaboticabal, 2009.

\begin{tabular}{|c|c|c|c|c|c|c|}
\hline \multirow{2}{*}{ Linhagens } & \multirow{2}{*}{ NTVC } & $M_{100}$ GV1G & $M_{100} G V 2 G$ & $M_{100}$ GV3G & MVNC & MV1G \\
\hline & & \multicolumn{3}{|c|}{ (g/100 grãos) } & \multicolumn{2}{|c|}{$\left(\mathrm{g} \mathrm{pl}^{-1}\right)$} \\
\hline BR36 & $100,96 \mathrm{a}$ & $41,23 \mathrm{~d}$ & $44,81 \mathrm{~d}$ & $45,65 \mathrm{~d}$ & $0,63 b$ & $11,96 \mathrm{a}$ \\
\hline JLM010 & $66,66 \mathrm{~b}$ & $82,65 \mathrm{a}$ & $90,40 \mathrm{a}$ & $90,49 a$ & $0,27 b$ & $25,86 \mathrm{a}$ \\
\hline JLM030 & $79,80 \mathrm{~b}$ & $60,59 \mathrm{~b}$ & $62,10 \mathrm{~b}$ & $62,31 \mathrm{~b}$ & $0,91 \mathrm{~b}$ & $15,14 \mathrm{a}$ \\
\hline JLM019 & $99,46 \mathrm{a}$ & $36,03 \mathrm{e}$ & $37,19 \mathrm{e}$ & $37,76 \mathrm{e}$ & $0,99 \mathrm{~b}$ & $6,00 \mathrm{a}$ \\
\hline BRS155 & $114,60 \mathrm{a}$ & $31,28 \mathrm{e}$ & $33,25 \mathrm{e}$ & $34,06 \mathrm{e}$ & $1,87 \mathrm{a}$ & $9,75 \mathrm{a}$ \\
\hline JLM018 & $88,83 \mathrm{a}$ & $41,87 \mathrm{~d}$ & $45,67 \mathrm{~d}$ & $42,02 \mathrm{~d}$ & $1,82 \mathrm{a}$ & $26,90 \mathrm{a}$ \\
\hline JLM003 & $70,83 \mathrm{~b}$ & $52,26 \mathrm{c}$ & $53,59 \mathrm{c}$ & $51,45 \mathrm{c}$ & $1,05 \mathrm{~b}$ & $18,50 \mathrm{a}$ \\
\hline JLM020 & $102,53 \mathrm{a}$ & $42,73 \mathrm{~d}$ & $43,81 \mathrm{~d}$ & $40,44 \mathrm{~d}$ & $2,35 \mathrm{a}$ & $32,56 \mathrm{a}$ \\
\hline JLM004 & $44,13 \mathrm{~b}$ & $56,55 \mathrm{~b}$ & $55,98 \mathrm{c}$ & $53,20 \mathrm{c}$ & $1,23 b$ & $13,61 \mathrm{a}$ \\
\hline JLM024 & $86,63 \mathrm{a}$ & $42,06 \mathrm{~d}$ & $43,31 \mathrm{~d}$ & $40,78 \mathrm{~d}$ & $1,01 \mathrm{~b}$ & $21,76 \mathrm{a}$ \\
\hline Teste $\mathrm{F}$ & $5,23 * *$ & $59,69 * *$ & $110,28 * *$ & $102,52 * *$ & $4,28 * *$ & $11,87^{\mathrm{NS}}$ \\
\hline CV (\%) & $18,44 \%$ & $6,88 \%$ & $5,27 \%$ & $6,68 \%$ & $43,02 \%$ & $33,34 \%$ \\
\hline
\end{tabular}

Médias seguidas pelas mesmas letras, na coluna, não diferem significativamente, em nível de 5\% de probabilidade, pelo teste de agrupa-

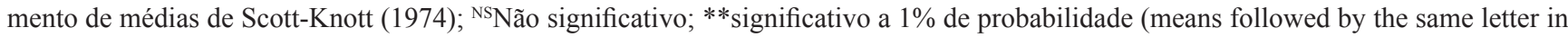
column are not different through the means grouping test of Scott-Knott (1974) at 5\%; NSNo significant; **significant at 1\% probability).

Tabela 3. Médias das características massa fresca de vagens com dois grãos (MV2G), massa fresca de vagens com três grãos (MV3G), massa fresca total de vagens comerciais (MTVC) e produtividade total estimada por hectare (PTEH) para dez linhagens de soja-hortaliça (average fresh weight of pods with two grains (MV2G), fresh weight of pods with three grains (MV3G), total fresh weight of marketable pods (MTVC) and estimated total yield per hectare for ten genotypes of vegetable soybean). UNESP, Jaboticabal, 2009.

\begin{tabular}{|c|c|c|c|c|}
\hline \multirow{2}{*}{ Linhagens } & MV2G & MV3G & MTVC & \multirow{2}{*}{ PTEH $\left(\mathrm{t} \mathrm{ha}^{-1}\right)$} \\
\hline & & $\left(\mathrm{g} \mathrm{pl}^{-1}\right)$ & & \\
\hline BR36 & $95,30 \mathrm{a}$ & $40,09 \mathrm{~b}$ & $147,51 \mathrm{a}$ & $9,99 \mathrm{a}$ \\
\hline JLM010 & $112,63 \mathrm{a}$ & $27,46 \mathrm{c}$ & $165,96 \mathrm{a}$ & $12,53 \mathrm{a}$ \\
\hline JLM030 & $109,55 \mathrm{a}$ & $28,80 \mathrm{c}$ & $153,50 \mathrm{a}$ & $10,48 \mathrm{a}$ \\
\hline JLM019 & $68,86 \mathrm{~b}$ & $52,11 \mathrm{a}$ & $126,99 \mathrm{~b}$ & $8,83 \mathrm{a}$ \\
\hline BRS155 & $54,58 \mathrm{~b}$ & $58,61 \mathrm{a}$ & $120,94 \mathrm{~b}$ & $9,07 \mathrm{a}$ \\
\hline JLM018 & $66,25 \mathrm{~b}$ & $7,30 \mathrm{~d}$ & $100,46 \mathrm{~b}$ & $6,95 \mathrm{~b}$ \\
\hline JLM003 & $77,64 b$ & $23,33 \mathrm{c}$ & $119,49 b$ & $7,48 \mathrm{~b}$ \\
\hline JLM020 & $79,86 \mathrm{~b}$ & $10,53 \mathrm{~d}$ & $122,96 \mathrm{~b}$ & $7,76 b$ \\
\hline JLM004 & $48,95 \mathrm{~b}$ & $13,36 \mathrm{~d}$ & $75,94 \mathrm{~b}$ & $4,55 \mathrm{c}$ \\
\hline JLM024 & $70,82 \mathrm{~b}$ & $25,70 \mathrm{c}$ & $118,30 \mathrm{~b}$ & $7,40 \mathrm{~b}$ \\
\hline Teste F & $5,47 * *$ & $14,15 * *$ & $4,58 * *$ & $6,56 * *$ \\
\hline CV $(\%)$ & $20,31 \%$ & $17,42 \%$ & $16,83 \%$ & $17,51 \%$ \\
\hline
\end{tabular}

Médias seguidas pelas mesmas letras, na coluna, não diferem significativamente, em nível de 5\% de probabilidade, pelo teste de agrupamento de médias de Scott-Knott (1974); ** significativo a $1 \%$ de probabilidade (means followed by the same letter in column are not different through the means grouping test of Scott-Knott (1974) at 5\%; NSNo significant; $* *$ significant at $1 \%$ probability). alto coeficiente de variação observado para esta característica $(33,34 \%)$, devido à dispersão dos dados experimentais para esta característica. Para massa fresca de vagens com dois grãos (Tabela 3) observou-se que a linhagem JLM010 se destacou $\left(112,63 \mathrm{~g} \mathrm{pl}^{-1}\right)$, porém não diferiu estatisticamente das linhagens BR36 (95,30 $\left.\mathrm{g} \mathrm{pl}^{-1}\right)$ e JLM030 (109,55 $\left.\mathrm{g} \mathrm{pl}^{-1}\right)$. As linhagens BRS155 e JLM019 se destacaram em relação à massa fresca de vagens com três grãos, com 58,61 e $52,11 \mathrm{~g} \mathrm{pl}^{-1}$ respectivamente.

As linhagens que apresentaram as maiores médias de massa fresca total de vagens comerciais foram, respectivamente, JLM010 (165,96 $\left.\mathrm{g} \mathrm{pl}^{-1}\right)$, JLM030 (153,50 $\left.\mathrm{g} \mathrm{pl}^{-1}\right)$ e BR36 (147,51 $\mathrm{g} \mathrm{pl}^{-1}$ ). Estes valores são superiores aos observados por Charlo et al. 2008 que ao avaliarem densidades de plantio de soja-hortaliça, verificaram massa fresca de vagens comerciais de $71,33 \mathrm{~g} \mathrm{pl}^{-1}$ para JLM010. Esta diferença entre os valores pode ser devido à época em que os experimentos foram conduzidos, 
visto que os experimentos dos referidos autores foram conduzidos de setembro a dezembro, e em ano diferente, portanto, pode ter havido influência das condições climáticas.

Para a produtividade total estimada de grãos imaturos, verificou-se que JLM010 conteve o maior valor $(12,53$ t ha-1), não diferindo estatisticamente das linhagens JLM030 (10,48 $\left.\mathrm{t} \mathrm{ha}^{-1}\right)$, BR36 (9,99 t ha-1), BRS155 (9,07 tha $\left.{ }^{-1}\right)$ e JLM019 (8,83 $\left.\mathrm{t} \mathrm{ha}^{-1}\right)$.

Estes valores são superiores aos obtidos no experimento de Smiderle et al. (2007), em que também separaram-se vagens com zero grão, um grão, dois grãos e três grãos, para posterior cálculo de produtividade, obtendo-se valores de produtividade variando de $3.447 \mathrm{~kg} \mathrm{ha}^{-1}$ (JLM 18) a $5.333 \mathrm{~kg} \mathrm{ha}^{-1}$ (JLM 08). Apesar da não separação de vagens com diferentes números de grãos para posterior cálculo da produtividade, no experimento de Castoldi et al. (2011), houve valores próximos aos do atual experimento, variando de 5,72 t ha-1 (JLM003) a 11,12 t ha $^{-1}$ (JLM010). Isto demonstra que a não separação de vagens não interfere na produtividade, porém é de fundamental importância no momento da comercialização, pois consumidores deste tipo de hortaliça são exigentes com relação à qualidade do produto, sendo que vagens com maior número de grãos, sem danificações e com grãos graúdos são mais atrativas aos consumidores.

Portanto, com base nas avaliações das principais linhagens com potencial de mercado para soja-hortaliça, nas condições deste experimento, pode-se concluir que JLM010 revelou melhor desempenho, por apresentar elevada produtividade e grãos graúdos.

\section{REFERÊNCIAS}

CARRÃO-PANIZZI MC. 2006. Edamame ou soja-hortaliça: fácil de consumir e muito saudável. Informe Agropecuário 27: 59-64.

CASTOLDI R; BRAZ LT; CHARLO HCO; CARRÃO-PANIZZI M C; MENDONÇA JL. 2008. Desempenho de genótipos de soja-hortaliça, em Jaboticabal-SP. Acta Horticulturae, 769:125-128.

CASTOLDI R; CHARLO HCO; VRGAS PF; BRAZ LT; CARRÃO-PANIZZI MC. 2011. Agronomic characteristics, isoflavone content and kunitz trypsin inhibitor of vegetable soybean genotypes. Horticultura Brasileira, 29: 222-227.

CHARLO HCO; CASTOLDI R; VARGAS PF; BRAZ LT; MENDONÇA JL. 2008. Desempenho de genótipos de soja-hortaliça de ciclo precoce [Glycine max (L.) Merril] em diferentes densidades. Ciência e Agrotecnologia, 32: 630-634.

COSTA JA; MARCHEZAN E. 1982. Características dos estádios de desenvolvimento da soja. Campinas: Fundação Cargill. 30 p.

Empresa Brasileira de Pesquisa Agropecuária. 1999. Centro Nacional de Pesquisa de Solos. Sistema brasileiro de classificação de solos: produção de informação. Rio de Janeiro: EMBRAPA. 412 p.

Empresa Brasileira de Pesquisa Agropecuária. 2003. Tecnologia de produção de soja - região central do Brasil 2004. Londrina: EMBRAPA. 237 p. (Sistemas de Produção, 4).

KONOVSKY J; LUMPKIN TA. 1990. Edamame production and use: a global perspective. In: INTERNATIONAL CONFERENCE SOYBEAN PROCESSING AND UTILIZATION, 4. Program and abstracts... Gonghuling: Jilin Academy of Agricultural Science (CD-ROM).

KURZER MS; XU X. 1997. Dietary phytoestrogens. Annual Review of Nutrition, 17: 353-381.

MENDONÇA JL; CARRÃO-PANIZZI MC. 2003. Soja-verde: uma nova opção de consumo. Brasília: Embrapa Hortaliças. 8 p. (Comunicado técnico).

MESSINA M. 2004. The Science behind soyafoods. In: WORLD SOYBEAN RESEARCH CONFERENCE, 7 . IN TERNATIONAL SOYBEAN PROCESSING AND UTILIZATION CONFERENCE, 4. BRAZILIAN SOYBEAN CONGRESS, 3. 2004, Foz do Iguaçu. Proceedings... Londrina: Embrapa Soja. p. 73-82.

RAIJ BV; CANTARELLA H; QUAGGIO JA; FURLANI AMC (ed). 1997. Recomendações da adubação e calagem para o Estado de São Paulo. 2. ed. Campinas: IAC. 285 p. (Boletim Técnico, 100).

SCOTT AJ; KNOTT MA. 1974. A cluster analysis method for grouping means in the analysis of variance. Biometrics 30: 507-512.

SHANMUGASUNDARAM S; YAN MR. 2004. Global expansion of high value vegetable soybean. In: WORLD SOYBEAN RESEARCH CONFERENCE, 7. INTERNATIONAL SOYBEAN PROCESSING AND UTILIZATION CONFERENCE, 4. BRAZILIAN SOYBEAN CONGRESS, 3. Proceedings... Londrina: Embrapa Soja. p. 915-920.

SMIDERLE OJ; GIANLUPPI V; SILVA SRG; SILVA JB. 2007. Produtividade e qualidade de sementes de genótipos de soja-hortaliça em cerrado de Roraima 2006/2007. In: REUNIÃO DE PESQUISA DE SOJA DA REGIÃO CENTRAL DO BRASIL, 24. Resumos... Londrina: Embrapa Soja. p. 159-161. 